\title{
Alar Batten Grafts for Non-iatrogenic Nasal Valve Area/Alar Collapse
}

\section{${ }^{1} \mathrm{C}$ Ekambar E Reddy, ${ }^{2}$ Trevor Teemul, ${ }^{3}$ Sucha Hampal, ${ }^{4}$ Krishna TV Reddy}

${ }^{1}$ Specialist Registrar, Department of Otolaryngology, Warrington Hospital, UK

${ }^{2}$ Senior House Officer, Department of Otolaryngology, Warrington Hospital, UK

${ }^{3,4}$ Consultant, Department of Otolaryngology, Warrington Hospital, UK

Correspondence: C Ekambar E Reddy, Specialist Registrar, Department of Otolaryngology, Warrington Hospital, Lovely Lane Warrington WA5 1QG, UK, Phone: +441925662034, Fax: +441925662042, e-mail: ekambarreddy@yahoo.com

\begin{abstract}
Objectives: We present our 3 years experience with alar batten grafts, using a modified technique, for non-iatrogenic nasal valve/alar collapse.

Methods: Retrospective (Oct. 2005 to Apr. 2008) and prospective study (Apr. 2008 to Dec. 2008) of 16 consecutive patients undergoing alar batten grafts for alar collapse causing nasal obstruction. The graft was inserted through a rim incision and placed across as well as superficial to the lower lateral cartilage. The main outcome measures were subjective improvement in nasal obstruction and absence of alar collapse at examination.

Results: Alar collapse was bilateral $(n=8)$, right sided $(n=4)$ and left sided $(n=4)$ giving 24 operated sides $(s=24)$. Deviated nasal septum $(n=9)$, synechiae $(n=1)$ and drooping tip $(n=1)$ were also present. Donor sites for the graft were septal cartilage $(n=10)$, conchal cartilage $(n=4)$ or both septal and conchal cartilages $(n=2)$. The median follow-up was 4 months (range 1 month to 3 years). Subjective nasal obstruction had worsened in $0 \%$, was unchanged in 16.6\% ( $s=4)$, had improved in $16.6 \%(s=4)$ and $66.6 \%(s=16)$ were completely free of obstruction. Two of the improved cases worsened at 10 and 3 years giving overall failure in $25 \%$ ( $s=6)$. Significant alar collapse was present in all failed cases and absence of collapse was documented in improved cases $(s=13)$. In failed cases the graft size width was 5 to $8 \mathrm{~mm}$ and in successful cases it was 10 to $15 \mathrm{~mm}$. Lengths of the grafts in all cases was between 18 and $24 \mathrm{~mm}$. One complication of graft extrusion through skin occurred.

Conclusion: Our technique of alar batten graft insertion appears to be as effective as other techniques described in the literature. We suggest a minimum graft size of $10 \mathrm{~mm}$ width and $18 \mathrm{~mm}$ length to improve success rate.
\end{abstract}

Keywords: Rhinoplasty, nasal valve collapse, cartilage graft.

\section{INTRODUCTION}

Nasal obstruction is one of the most common presenting complaints in otolaryngology clinics. Nasal obstruction is a complex problem, caused not just by deviated nasal septum but can have a long differential diagnosis, often is multifactorial. ${ }^{1,2}$ One of the causes of nasal obstruction is nasal valve area collapse. Nasal valve collapse is a neglected and often unrecognized entity.

Nose has an internal and an external nasal valve anatomically. ${ }^{3,4}$ The internal nasal valve is formed by the junction of the scroll region (area where lower lateral cartilage overlaps the upper lateral cartilage) and septum. External nasal valve is the caudal margin of the nostril itself. Nasal sidewall collapse is a preferred term and we have specifically studied only cases with nasal alar collapsing. Most common cause of nasal valve dysfunction is iatrogenic following a rhinoplasty. It can also occur due to trauma or idiopathic weakness of lower or upper lateral cartilages of the nose. Non-iatrogenic cases of alar collapse is seen in older patients as ageing can weaken the fibroareolar tissues of the nasal sidewall leading to its sagging and collapse on inspiration.

There are various ways of managing nasal sidewall/alar collapse (NAC) including spreader grafts, butterfly grafts, alar batten grafts (ABG), flare sutures and suspension sutures. ${ }^{5}$ Conservative measures like nasal breathing strips and Francis alar dilator have also been used. ABG strengthens and supports the weak area to prevent collapse on inspiration. Majority of the alar batten grafts are inserted via an inter cartilaginous incision and placed lateral to the lower lateral cartilage. ${ }^{4-6}$ We present our 3 years experience with alar batten grafts, using a modified technique, for noniatrogenic nasal valve area weakness. This is to report mainly our short-term (and in some cases medium-term) functional results and cosmetic sequelae. 


\section{METHODS}

This study was an audit of the data collected on 16 consecutive patients undergoing ABG for NAC causing nasal obstruction from Oct. 2005 to Dec. 2008 at a District Hospital in UK. Case notes were reviewed for the patients operated from Oct. 2005 to Apr. $2008(n=11)$ and data was prospectively collected for those operated between Apr 2008 and Dec. $2008(n=5)$. Only patients with nasal obstruction caused by NAC which improved with modified Cottle's maneuver (Jobson Horne wax probe was used to retract the nasal sidewall laterally for approximately 1 to $2 \mathrm{~mm}$ ) had ABG in our hospital. ABGs used in postrhinoplasty cases $(n=5)$ and to support alar reconstruction after skin cancer excisions $(n=3)$ were excluded. Patients with coexisting nasal polyps or allergic rhinitis were also excluded. The median age of the patients was 57.5 years (range of 18 to 69 years). Most of the patients were males $(n=14)$.

The surgery was performed under general anesthesia. Before the patient was put to sleep area of NAC was marked and 2\% lignocaine with 1: 80,000 adrenaline injected. Graft was harvested either from the septum or conchal cartilage or both. Conchal cartilage was the donor cartilage when septal cartilage was insufficient or when septoplasty was done in the past. Marginal/rim incision, just long enough to insert the graft, was made along the lower border of the lateral crura of the lower lateral cartilage (Fig. 1A). Precise pocket was made, in the marked area, just superficial to the lower lateral cartilage extending on the pyriform aperture superiorly and towards the nostril rim inferiorly. The graft was placed perpendicular to the orientation of the lower lateral cartilage extending onto the bone of the pyriform aperture (Fig. 1B). Incision was closed with 40 vicryl. No packs were inserted and patient was sent home same day. Dimensions of the ABG were noted.

The main outcome measures were subjective improvement in nasal obstruction postoperatively as expressed by the patients (vastly improved/absence of nasal obstruction; improved; unchanged; or worsened) and absence of NAC at examination. Secondary outcome measure (cosmetic) studied was patient's complaint about bulky nose or palpating a prominent graft and obliteration of supra-alar crease at examination. The patients were reviewed at 6 weeks and 3 months in the prospective group. Occurrence of any complications was also noted.

Ethical approval was not needed as this study was an audit of our current practice.

\section{RESULTS}

All the patients presented with nasal obstruction and 3 patients also complained of snoring. Some patients $(n=8)$

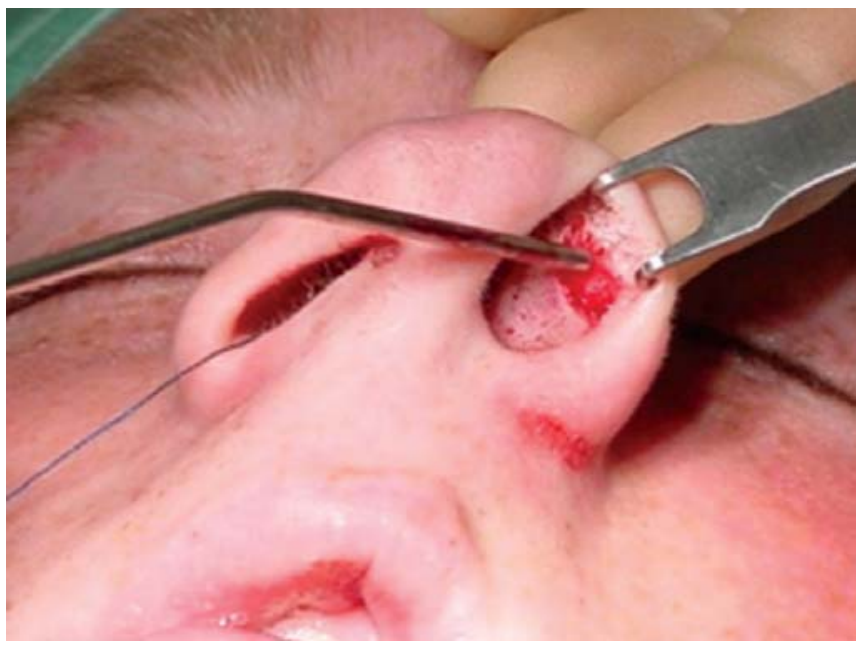

Figure 1A: Shows the rim incision

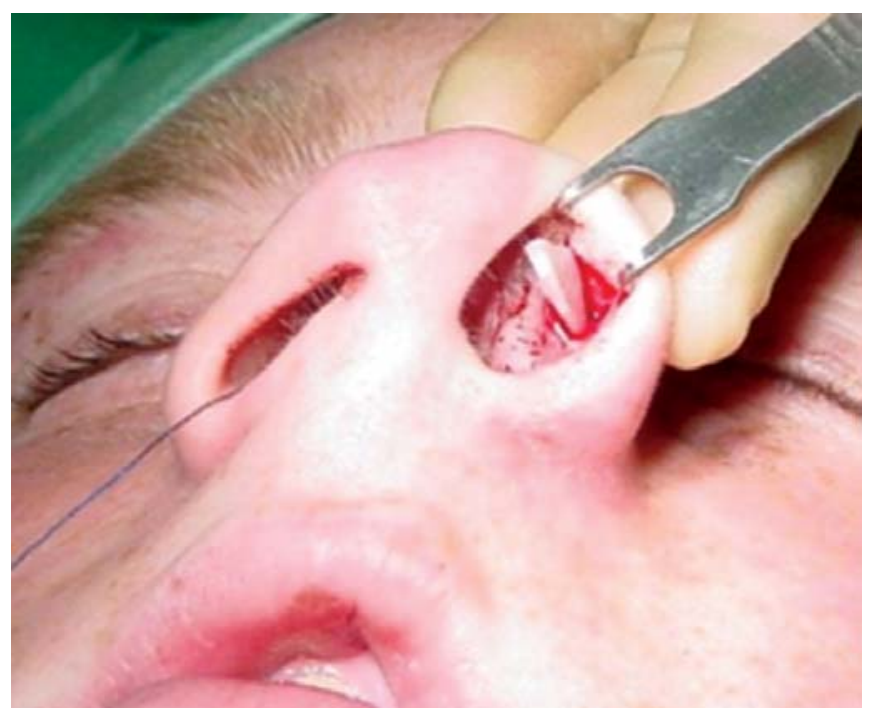

Figure 1B: Shows our technique of inserting the graft

had underwent nasal surgeries in the past. These were septoplasty $(n=2)$, septoplasty with inferior turbinectomy $(\mathrm{n}=1)$, septoplasty with functional endoscopic sinus surgery for sinusitis ( $n=1)$, endoscopic sinus surgery for polyps $(n=1)$ and manipulation of the nasal bones $(n=1)$. There was history of trauma to the nose in 3 patients.

NAC was bilateral $(n=8)$, right sided $(n=4)$ and left sided $(n=4)($ Fig. $2 A)$ giving 24 operated sides $(s=24)$. Deviated nasal septum (DNS) $(\mathrm{n}=9)$, synechiae $(\mathrm{n}=1)$ and drooping tip $(\mathrm{n}=1)$ were the other signs noted during examination. DNS in unilateral cases $(n=6)$ was to opposite side of NAC $(n=3)$, same side of NAC $(n=2)$ and ' $S$ ' shaped $(n=1)$. The rest of the patients with DNS $(n=3)$, presence of unilateral synechiae and drooping tip were in cases with bilateral NAC. Septoplasty and synechiae division was also done in these patients. 


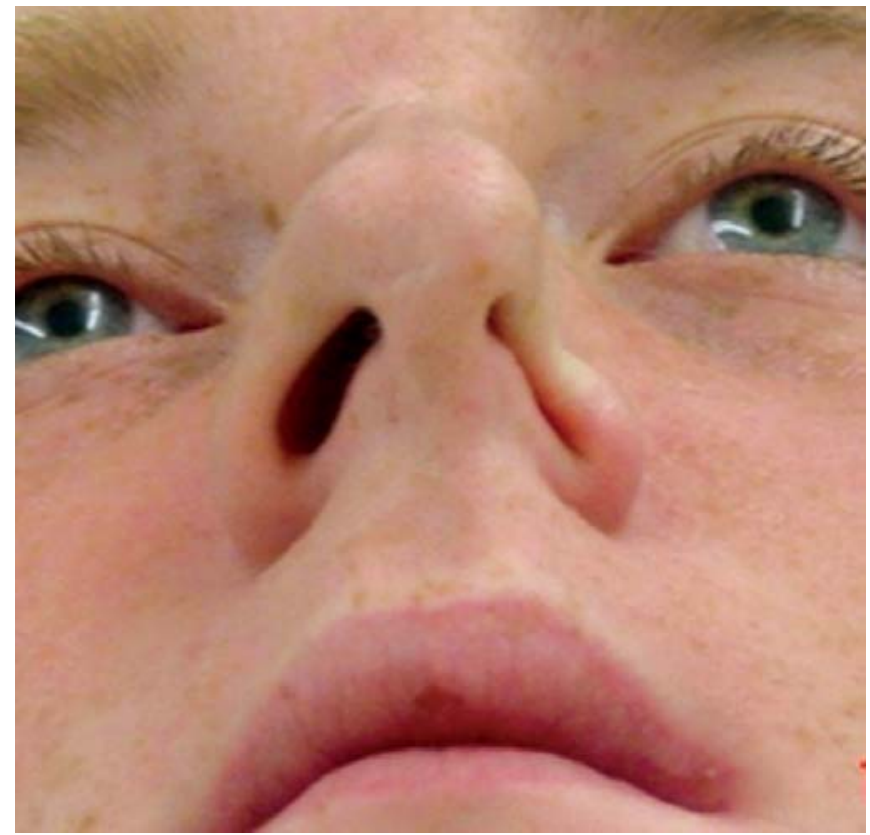

Figure 2A: Shows left alar collapse on inspiration preoperatively

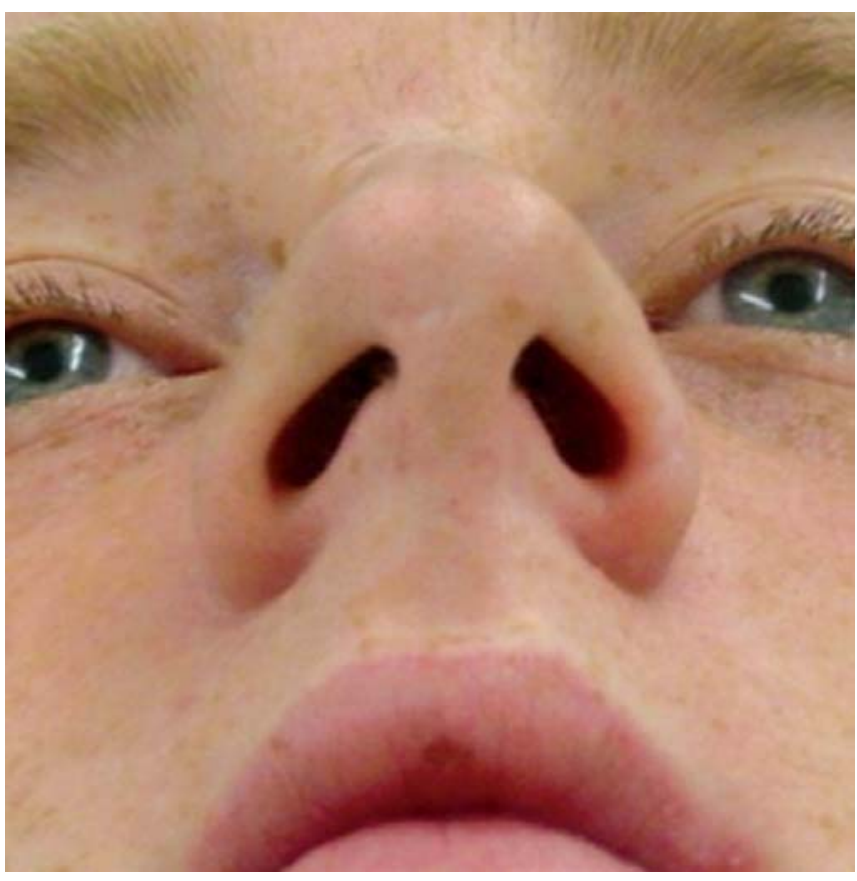

Figure 2B: Shows absence of alar collapse after surgery

We used autologous cartilage as ABG in all the patients. Donor sites for the graft were septal cartilage $(n=10)$, conchal cartilage $(n=4)$ or both septal and conchal cartilages $(\mathrm{n}=2)$. The graft dimensions were accurately recorded in the all 5 cases whose data was collected prospectively $(s=9)$. In the retrospective cases 6 had data on dimensions $(s=10)$.
The median follow-up was 4 months (range 1 month to 3 years). Subjective nasal obstruction had worsened in $0 \%$, was unchanged in $16.6 \%(\mathrm{~s}=4)$, was improved in $16.6 \%$ $(\mathrm{s}=4)$ and $66.6 \%(\mathrm{~s}=16)$ were completely free of obstruction. Two unilateral improved patients presented back at 10 months and 3 years respectively with worsening of the improved symptoms. Overall short-term functional improvement was $83.3 \%(\mathrm{~s}=20)$ but deteriorated over mid-term to $75 \%(s=18)$ of the sides operated. Patients with bilateral NAC $(\mathrm{n}=8)$ had improvement on both sides in $62.5 \%(n=5)$, one side in $25 \%(n=2)$ and no improvement on both sides in $12.5 \%(\mathrm{n}=1)$. All 8 patients with unilateral NAC had improvement (100\%) in the short-term and in 2 it failed later (25\%). All patients with DNS had improvement in short-term and 2 patients later worsened $(n=2 ; s=2)$. Overall success in cases with DNS ( $\mathrm{n}=7 ; \mathrm{s}=10)$ was $77.8 \%$ of patients and $83.3 \%$ of sides.

Significant NAC was present in all failed cases and absence of collapse on inspiration (Fig. 2B) was documented in improved cases $(s=13)$. In failed cases the graft size width was 5 to $8 \mathrm{~mm}$ and in successful cases it was between 10 and $15 \mathrm{~mm}$. Lengths of the grafts in all cases was between 18 and $24 \mathrm{~mm}$.

Cosmetic concerns were expressed by two patients only but all 5 prospectively studied cases showed some obliteration of supra-alar crease which was not noticed by the patients. One complication of graft extrusion through dorsal skin occurred which was removed and it healed very well.

\section{DISCUSSION}

NAC can be missed unless specifically sought. The purpose of treating nasal valve area weakness is to prevent inspiratory collapse while avoiding deleterious cosmetic sequelae. We highlight our technique of ABG with good if not excellent functional outcome. Untoward cosmetic effects and complications were rare.

This study has limitations like any other retrospective study. There was incomplete data on the cosmetic outcome and alar batten graft size. DNS was also corrected in 9 cases and this could be a factor affecting the main outcome. Subjective improvement of airway was better in these patients than those without DNS. We feel that our main outcome measure although subjective is a pragmatic approach for auditing our current practice. More objective measure of the functional outcome can be done using anterior active mask rhinomanometry. ${ }^{2}$

Most of the studies reporting ABG use are for postrhinoplasty nasal valve collapse. ${ }^{2,4,6-10}$ Our study exclusively reports non-iatrogenic nasal valve area collapse. The results 
of combination techniques for dysfunctional nasal sidewall are described. ${ }^{2,7,9,10}$ After thorough literature search we could find only 3 articles in the English literature solely devoted to ABG. ${ }^{4,6,8}$ Millman $B^{4}$ operated on 21 patients only one of whom had a previous rhinoplasty and report $100 \%$ improvement at 12 months. All patients were pleased with their esthetic result, but in 6 patients the authors noticed fullness in the scroll region. Forty-six patients surveyed by Toriumi DM et al ${ }^{6}$ had mean improvement of nasal airway by 2.5 on a scale of 5 in all but one patient. They neither mention about patient demographics nor previous operations nor the cause of NAC. They report minimal fullness in the supra-alar region in 'some' cases. Becker DG et $\mathrm{al}^{8}$ report excellent results where ABG were used with septoplasty or septorhinoplasty. Our results are comparable to some of these studies. None of the studies describe graft dimensions.

All the authors except Becker DG ${ }^{3}$ insert the graft via inter cartilaginous incision and graft is oriented horizontally along alar rim. We do it via rim incision and orientate the graft obliquely to lie cranially over the pyriform aperture. We feel this strengthens more area of nasal sidewall. Our technique of making a precise pocket for the graft avoids the need for suture fixation. Inserting the lower margin of the graft in the pocket distal to the incision more or less eliminates extrusion through the incision.

\section{CONCLUSION}

Our technique of alar batten graft insertion appears to be as effective as other techniques described in the literature.
We suggest a minimum graft size of $10 \mathrm{~mm}$ width and 18 $\mathrm{mm}$ length to improve the success rate. Further studies with measurement of graft dimensions are needed to confirm or refute our findings.

\section{REFERENCES}

1. Teichgraeber JF, Wainwright DJ. The treatment of nasal valve obstruction. Plast Reconstr Surg 1994;93:1174-82.

2. Constantian MB, Clardy RB. The relative importance of septal and nasal valvular surgery in correcting airway obstruction in primary and secondary rhinoplasty. Plast Reconstr Surg 1996;98:38-54.

3. Murakami C. Nasal valve collapse. Ear Nose Throat J 2004;83:163-64.

4. Millman B. Alar batten grafting for management of the collapsed nasal valve. Laryngoscope 2002;112:574-79.

5. Wittkopf M, Wittkopf J, Ries WR. The diagnosis and treatment of nasal valve collapse. Curr Opin Otolaryngol Head Neck Surg 2008;16:10-13.

6. Toriumi DM, Josen J, Weinberger M, Tardy ME. Use of alar batten grafts for correction of nasal valve collapse. Arch Otolaryngol Head Neck Surg 1997;123:802-08.

7. Stucker FJ, Hoasjoe DK. Nasal reconstruction with conchal cartilage: Correcting nasal valve and lateral nasal collapse. Arch Otolaryngol Head-Neck Surg 1994;120:653-8.

8. Becker DG, Becker SS. Treatment of nasal obstruction from nasal valve collapse with alar batten grafts. J Long Term Eff Med Implants 2003;13:259-69.

9. Goode RL. Surgery of the incompetent nasal valve. Laryngoscope 1985;95:546-55.

10. Ballert JA, Park SS. Functional rhinoplasty: Treatment of dysfunctional nasal sidewall. Facial Plast Surg 2006;22:49-54. 